\title{
Theoretical Modelling of Wakes from Retractable Flapping Wings in Forward Flight
}

A free-wake method is used to simulate the wake from retractable, jointed wings. The method serves to complement existing experimental studies that visualise flying animal wakes. Simulated wakes are shown to be numerically convergent for a case study of the Rock Pigeon in minimum power cruising flight. The free-wake model is robust in simulating wakes for a range of wing geometries and dynamics without requiring changes to the numerical method. The method is found to be useful for

providing low order predictions of wake geometries. However, it is not well suited to reconstructing 3d flowfields as solutions are sensitive to the numerical mesh node locations. 
1 Dr Ben Parslew*, Dr William J. Crowther

2 School of Mechanical, Aerospace and Civil Engineering, The University of Manchester, PO Box 88,

3 M60 1QD, UK

4 *Corresponding author. Tel: +44 161 3063755. E-mail address: ben.parslew@manchester.ac.uk

\section{1. Introduction}

6 Fluidic forces on flying animals have been derived from direct measurements of pressure on the wing

7 and tail [1],[2]. Flow visualisation is an alternative technique that allows forces to be inferred through

8 examining the structure of the wake (e.g. [3], [4], [5]) this approach aims to reduce interference

9 between the subject and measurement apparatus, allowing the experimenter to capture more natural

10 kinematics.

11 To complement experimental measurements, theoretical models of wakes have been developed that

12 predict flying performance. Early examples include models of flapping-wing flight that prescribe the

13 wake geometry as a series of vortex rings [6], [7], [8], [9]. However, prescribed-wake models are

14 limited by their inability to capture evolution of the wake geometry over time. More recently,

15 Navier-Stokes computations have been used to simulate the flow around flapping wings (e.g. [10]).

16 While this approach calculates properties of the entire flowfield, there are drawbacks in terms of

17 computational cost. Also, considerable effort is required to construct the numerical mesh that

18 discretizes the flowfield. 
19 Free-wake methods developed for aerospace applications simulate wakes from lifting surfaces without

20 resolving the entire flowfield [11], [12], [13], [14], [15]). These methods calculate induced velocity

21 from vorticity in the wake, which arises due to the presence of a lifting surface. As a result, they

22 implicitly predict the evolution of the wake geometry as it propagates downstream. Free-wake models

23 are not restricted to specific lifting surface kinematics, and are therefore applicable to fixed, rotary

24 and flapping wings.

25 The contribution of this paper is to apply the free-wake methodology to retractable, jointed, flapping

26 wings. A parsimonious approach will be used, whereby a simple but extensible model will be

27 developed using a minimal number of input parameters. The model will capture the fundamental wake

28 geometry, and can later be extended to simulate more complex fluidic phenomena such as vortex

29 viscous core growth and vortex stretching. The primary aim of the study is to demonstrate numerical

30 convergence of the model for a case study of the Rock Pigeon, Columbia livia, in cruising flight;

31 numerical convergence will be recognised as the tendency of the simulation to approach a fixed

32 solution as the resolution of the numerical scheme increases. Preliminary findings for simulated wake

33 geometries will be presented for different wing geometries and dynamics.

\section{2. Method}

\section{2.1. Free-wake model}

36 The free-wake model presented here is derived from a model used for analysing helicopter rotor

37 wakes [13][16]. Vortex filaments are assumed to be shed from the trailing edge of the wing (figure 1).

38 Filaments are discretized into a series of segments between Lagrangian markers. Markers move with

39 the local flow velocity, transporting flow properties with them. Modelling the flow as inviscid and

40 irrotational, Helmholtz's second law of vortex motion implies that vortex filaments move as material

41 lines, and thus the velocity of any marker is equal to the local flow velocity: 
$\frac{u s}{1}=V$

42

$43 \mathbf{x}$ is the marker position and $\mathbf{V}$ is the local fluid velocity, which is the vector sum of the freestream

44 velocity, $\mathbf{V}_{\infty}$ (due to motion of the body through the fluid), and the velocity induced from vortex

45 filaments, $\mathbf{V}_{\mathrm{I}}$. The main challenge in solving equation (1) is calculating the vortex induced velocity.

46 This is achieved using the Biot-Savart law, which forms the foundation of the model (section 2.2.).

47 The free-wake model can be implemented using three different approaches (figure 1; a-c). Horseshoe

48 vortex models assume a bound vortex on the wing and a pair of trailing vortex filaments shed from

49 the trailing edge. The simplest model assumes a single horseshoe vortex, with trailing filaments shed

50 from the wingtips (figure 1; a). Multiple horseshoe vortices can be used to model the effects of

51 non-uniform lift distributions on the wing that occur during flapping-wing flight (figure 1; b).

52 The vortex ring model (figure 1; c) is similar to the trailing vortex model, but also takes into account

53 spanwise vorticity shed due to temporal changes in lift. Shed vortex segments are calculated as line

54 segments between markers shed at the same time on adjacent filaments. Circulation of these segments

55 is given as the change in bound circulation on the wing over the time interval between the release of

56 successive markers [14]. The circulation on a segment between a pair of markers is the vector sum of

57 the circulation from the two adjacent vortex rings on which the markers lie (figure 2; b). The vortex

58 ring model is selected for the present study to capture spanwise vorticity that arises in flapping-wing

59 wakes due to the temporal variation in lift throughout the wingbeat.

\section{2.2. Induced velocity}

61 Velocity induced on a marker located at $\mathbf{x}_{\mathrm{m}}$ due to the presence of the $n$th filament segment is given

62 from the Biot-Savart law: 


$$
\mathbf{V}_{I}=\frac{1 \mathbf{u} \wedge\left|\mathbf{\Lambda}_{m}-\mathbf{A}\right|}{4 \pi \|\left(\mathbf{x}_{m}-\left.\mathbf{x}^{(n)}\right|^{3} \|\right.}
$$

64 where $\Gamma^{(\mathrm{n})}$ and $\mathbf{d}^{(\mathrm{n})}$ are the circulation and length of the $n$th filament segment and $\mathbf{x}^{(\mathrm{n})}$ is the location of

65 the $n$th segment marker. Typically, a vortex core model is used to avoid numerical singularities when

66 the distance between the marker and filament segment tends to zero [15]. However, in the present

67 work all simulations were found to be stable and convergent without using a viscous vortex core

68 model.

69 The contribution of induced velocity from each filament segment is calculated using equation (2) and

70 summed to give the net velocity on a marker. The procedure is repeated for all markers, forming an

$71 n$-body problem. This method of direct evaluation is only feasible for small numbers of markers and

72 could be accelerated by long-range cutoff approximations [15]; as the present algorithm has not been

73 optimised for performance the computational cost increases quadratically with the number of markers,

74 and is not representative of the cost of most point vortex simulations.

75 The local lift per unit span on a surface, $L$ ', is calculated using blade-element theory [12], [16]; while

76 this approach only captures quasi-steady aerodynamic lift the wake model can also be used with

77 methods that include unsteady effects. The Kutta-Joukowski theorem gives the circulation around the

78 wing as

$$
\Gamma=\frac{-}{{ }_{n}\|\mathbf{V}\|}
$$

79

(3). 
80 The instantaneous circulation is calculated for each blade-element, giving the shed and trailing

81 circulation of filament segments shed from the trailing edge at a given time. Equation (2) is then

82 evaluated for all markers in a time-marching simulation. Marker locations are updated using a

83 one-step Euler integration scheme for the first timestep, and a two-step Adams-Bashforth scheme for

84 all subsequent timesteps.

\section{2.3. Limitations}

86

87

88

89

90

91

92

93

94

95

96

97

98

99

100

101

102
- Induced velocity predicted by the free-wake simulation is not included in the blade-element model. As the freestream velocity magnitude is typically ten times that of the induced velocity, this approximation is not believed to significantly affect the simulated wake geometry.

- The Kutta condition is not enforced in the model, meaning that the velocity field immediately downstream of the lifting surface trailing edge will not be accurately resolved. This factor will be most significant when the angle attack is high, such as in slow flight and hover. Despite this, the model is still useful for making low-order predictions of the wake geometry as it propagates downstream.

- As an inviscid flow model, the wake dynamics are driven purely by circulation due to lift. Other force components on the wing have no influence on the wake. Therefore the model is not appropriate for simulating wakes behind bluff bodies, for example, where body forces are dominated by drag. Also, the inviscid flow model is appropriate for high Reynolds number flows such as those past large birds, but will be less accurate for modelling smaller flapping-wing animals, such as insects, where viscous effects become dominant.

- As vortex stretching is neglected, the model will not capture the increase in vorticity that arises on strained vortex filament segments [17]. 


\section{Results}

104 For a pair of fixed, rectangular wings the wake rolls up downstream of both the inboard and outboard

105 sections, as each wing acts as a separate lifting surface (figure 3; a). For flying animals lift

106 augmentation from the body would reduce the strength of trailing vorticity in the inboard sections.

107 This phenomenon is readily modelled using the existing method by setting the circulation of the most

108 inboard filaments to zero, which implicitly defines a uniform lift distribution across the body where

109 the lift per unit span is equal to that of the wings (figure 3; b). The geometry of the wake is visualised

110 more clearly by applying a cubic spline interpolation of the marker positions (figure 3; c).

111 A numerical convergence study for flapping-wing wakes was conducted by varying the three wake

112 parameters: the number of timesteps per cycle, $n_{t}$, number of vortex filaments, $n_{f}$, and number of

113 markers released per cycle for each filament, $n_{m}$. The normalised marker position, $p_{\text {norm }}$, was used to

114 measure the overall wake geometry:

$$
p_{\text {norm }}=\frac{L\|\|^{\prime}{ }^{\prime} \|}{n_{n}\|\mathbf{v}\|_{T}}
$$

116 where is the distance travelled by the body over the period, $T$. Wing dynamics were taken from

117 a model of the Rock Pigeon in minimum power cruising flight (cruising at $12 \mathrm{~ms}^{-1}$, with a chord-based

118 Reynolds number of $10^{4}$ ) [12]. Circulation of the inboard filaments was again set to zero to assume a

119 uniform lift distribution on the body. The wake was simulated for three wingbeats, and $p_{\text {norm }}$ was

120 derived for markers released during the third wingbeat. Extending the simulation to four wingbeats

121 led to less than $1 \%$ change in $p_{\text {norm }}$. As with previous free-wake models [12] the wake geometry

122 converged when increasing the three wake parameters (figure 4). Doubling any of the three

123 parameters from the maximum values shown in figure 4 led to less than $1 \%$ change in $p_{\text {norm }}$. 
124 As expected from the lift distribution on a flapping wing, the wake rolls up downstream of the wing

125 tips where the circulation strength is highest (figure 5). The decrease in lift from the wing tip to wing

126 root gives negative circulation on the inboard trailing filaments. Thus, the wake rollup downstream of

127 the inboard wing is of the opposite sense to the rollup downstream of the wing tip.

128 The wake geometry continues to evolve due to the self-induced velocity as it propagates downstream.

129 This highlights physical instability in the wake [18], and is not the result of errors in the numerical

130 method. Interaction between starting and tip vortices can be observed in the wake shed during the first

131 wingbeat (figure 5; c). This demonstrates the need to simulate the wake for more than one wingbeat to

132 reduce the influence of the starting vortex on the overall wake geometry and thus obtain a steady-state

133 solution.

134 Induced velocity can also be calculated on a mesh of nodes at fixed locations (figure 5; d). This

135 velocity field illustrates the counter-rotating wake regions downstream of the wing tips and roots, as

136 seen in previous experimental studies of flying animal wakes [19],[20],[21],[22],[23],[24],[25].

137 However, as vorticity is distributed on a discrete number of markers, reconstructed fields contain

138 fluctuations in regions where the grid nodes are near to the markers. This led to the predicted induced

139 velocity magnitudes being sensitive to the choice of plane streamwise location. For this reason the

140 authors have chosen not to attempt to compare the computed velocity fields with those measured in

141 previous flow visualisation experiments as this would be potentially misleading.

\section{4. Discussion}

143 The free-wake method is robust, in that it can simulate wakes for different wing geometries and

144 dynamics without requiring any changes to the underlying method. However, for extreme wing

145 retraction a vortex core model is necessary to avoid numerical instabilities [12]; when simulating the

146 wake for cruising flight of the pigeon numerical instabilities were observed when the wing length on

147 the upstroke was reduced to less than a quarter of its outstretched length. 
148 Simulated fixed-wing wakes illustrated the need to define an appropriate lift distribution on the body

149 to accurately predict the wake geometry downstream of the inboard wing. Conversely, flapping-wing

150 wake geometries were insensitive to the model of body lift; wakes simulated with uniform or zero lift

151 per unit span on the body contained no discernible differences in geometry. This is because the wake

152 geometry is dominated by the rollup from the wing tip vortices, inboard wing vortices, and starting

153 vortex, which occur for both models of body lift.

154 The starting vortex rollup caused significant differences in the wake geometry during the first

155 wingbeat when compared to previous simulations that used a multiple horseshoe vortex model [12].

156 Despite this, wake geometries reached periodic solutions after three wingbeats. The computation time

157 required to simulate the vortex ring model is significantly greater than for horseshoe vortex models, as

158 the Biot-Savart law is evaluated for both trailing and shed vortex segments.

159 The free-wake method is useful for providing low order predictions of wake geometries for different

160 wing geometries and dynamics. However, reconstructing the velocity field is more challenging and

161 should be approached with some caution. Modelling the viscous vortex core has potential for

162 preventing fluctuations in the induced velocity field, but this introduces additional uncertainty into the

163 model in defining an appropriate vortex core radius.

\section{Acknowledgement}

165 This work was funded by the Engineering and Physical Sciences Research Council (EPSRC) through

166 the Postdoctoral Fellowship Prize. 
168 [1] J. R. Usherwood, T. L. Hedrick, and A. A. Biewener, 'The aerodynamics of avian take-off from direct pressure measurements in Canada geese (Branta canadensis)', The Journal of Experimental Biology, vol. 206, pp. 4051-4056, 2003.

[2] J. R. Usherwood, T. L. Hedrick, C. P. McGowan, and A. A. Biewener, 'Dynamic pressure maps for wings and tails of pigeons in slow, flapping flight, and their energetic implications', The Journal of Experimental Biology, vol. 208, pp. 355-369, 2005.

174 [3] G. Lauder and E. Drucker, 'Forces, fishes, and fluids: hydrodynamic mechanisms of aquatic locomotion’, News in Physiological Sciences, vol. 17, pp. 235-240, 2002.

[4] G. R. Spedding and A. Hedenström, 'PIV-based investigations of animal flight', Experiments in Fluids, vol. 46, pp. 749-763, 2008.

178 [5] G. K. Taylor, M. S. Triantafyllou, and C. Tropea, Animal Locomotion, 1st Edition. Springer, 2010.

[6] J. M. V. Rayner, 'A vortex theory of animal flight. Part 1. The vortex wake of a hovering animal’, Journal of Fluid Mechanics, vol. 91, pp. 697-730, 1979.

182 [7] J. M. V. Rayner, 'A new approach to animal flight mechanics', The Journal of Experimental Biology, vol. 80, pp. $17-54,1979$.

184 [8] J. M. V. Rayner, 'Vertebrate flapping flight mechanics and aerodynamics, and the evolution of flight in bats', in Biona report 5, bat flight-fledermausflug, Gustav Fischer Verlag, 1987, pp. $27-74$. 
[9] C. P. Ellington, 'The Aerodynamics of Hovering Insect Flight. V. A Vortex Theory', Philosophical Transactions of the Royal Society of London. Series B, Biological Sciences, vol. 305, no. 1122, pp. 115-144, Feb. 1984.

[10] H. Liu, C. Ellington, K. Kawachi, C. van der Berg, and A. Willmott, ‘A computational fluid dynamic study of hawkmoth hovering', Journal of Experimental Biology, 1998.

[11] Smith, Wilkin, and Williams, 'The advantages of an unsteady panel method in modelling the aerodynamic forces on rigid flapping wings', The Journal of Experimental Biology, vol. 199, no. Pt 5, pp. 1073-1083, 1996.

[12] B. Parslew, 'Simulating Avian Wingbeats and Wakes', PhD Thesis, The University of Manchester, 2012.

[13] A. Bagai and J. Leishman, 'Rotor free-wake modeling using a pseudo-implicit technique including comparisons with experiment', Journal of the American Helicopter Society, vol. 40, pp. 29-41, 1995.

[14] M. J. Tarascio, M. Ramasamy, I. Chopra, J. G. Leishman, P. B. Martin, E. Smith, Y. H. Yu, A. P. F. Bernhard, and D. J. Pines, ‘Flow Visualization of Micro Air Vehicle Scaled Insect-Based Flapping Wings.', Journal of Aircraft, vol. 42, no. 2, pp. 385-390, Mar. 2005.

[15] M. Stock, 'A regularized inviscid vortex sheet method for three dimensional flows with density interfaces’, PhD dissertation, University of Michigan, 2006.

[16] B. Parslew and W. J. Crowther, 'Simulating avian wingbeat kinematics', Journal of Biomechanics, vol. 43, pp. 3191-3198, 2010.

[17] S. Ananthan and J. G. Leishman, 'Role of filament strain in the free-vortex modeling of rotor wakes’, Journal of the American Helicopter Society, vol. 49, pp. 176-191, 2004. 
[18] R. J. Bomphrey, P. Henningsson, D. Michaelis, and D. Hollis, 'Tomographic particle image velocimetry of desert locust wakes: instantaneous volumes combine to reveal hidden vortex elements and rapid wake deformation', J R Soc Interface, vol. 9, no. 77, pp. 3378-3386, Dec. 2012.

[19] P. Henningsson, F. T. Muijres, and A. Hedenström, 'Time-resolved vortex wake of a common swift flying over a range of flight speeds', Journal of The Royal Society Interface, vol. 8, pp. 807-816, 2011.

[20] A. Hedenström, L. C. Johansson, M. Wolf, R. von Busse, Y. Winter, and G. R. Spedding, 'Bat Flight Generates Complex Aerodynamic Tracks’, Science, vol. 316, no. 5826, pp. 894-897, 2007.

[21] F. T. Muijres, L. C. Johansson, R. Barfield, M. Wolf, G. R. Spedding, and A. Hedenström, ‘Leading-Edge Vortex Improves Lift in Slow-Flying Bats’, Science, vol. 319, no. 5867, pp. 1250-1253, 2008.

[22] T. Y. Hubel, D. K. Riskin, S. M. Swartz, and K. S. Breuer, 'Wake structure and wing kinematics: the flight of the lesser dog-faced fruit bat, Cynopterus brachyotis', J Exp Biol, vol. 213, no. 20, pp. 3427-3440, 2010.

[23] A. Hedenström, F. Muijres, R. von Busse, L. Johansson, Y. Winter, and G. Spedding, 'High-speed stereo DPIV measurement of wakes of two bat species flying freely in a wind tunnel', Experiments in Fluids, vol. 46, no. 5, pp. 923-932, May 2009.

[24] L. C. Johansson and A. Hedenstrom, 'The vortex wake of blackcaps (Sylvia atricapilla L.) measured using high-speed digital particle image velocimetry (DPIV)', The Journal of Experimental Biology, vol. 212, pp. 3365-3376, 2009. 
231 [25] R. J. Bomphrey, G. K. Taylor, and A. L. R. Thomas, 'Smoke visualization of free-flying

232 bumblebees indicates independent leading-edge vortices on each wing pair', Experiments in

$233 \quad$ Fluids, vol. 46, no. 5, pp. 811-821, Apr. 2009.

234 


\section{Figure 1}

Free-wake models

(a) Single horseshoe vortex model; (b) multiple horseshoe vortices model; (c) vortex ring model. Horseshoe vortex models include the bound vorticity on the lifting surface and trailing vorticity in the wake. In all models the wake downstream of the wingtips rolls up, and this is visible in models (b) and (c) as the trailing vortex filaments twist. The vortex ring model (c) also includes the transverse vorticity component that is shed from the surface due to temporal changes in lift; when the surface is accelerated from rest the transverse vorticity component leads to a starting vortex. 
a)

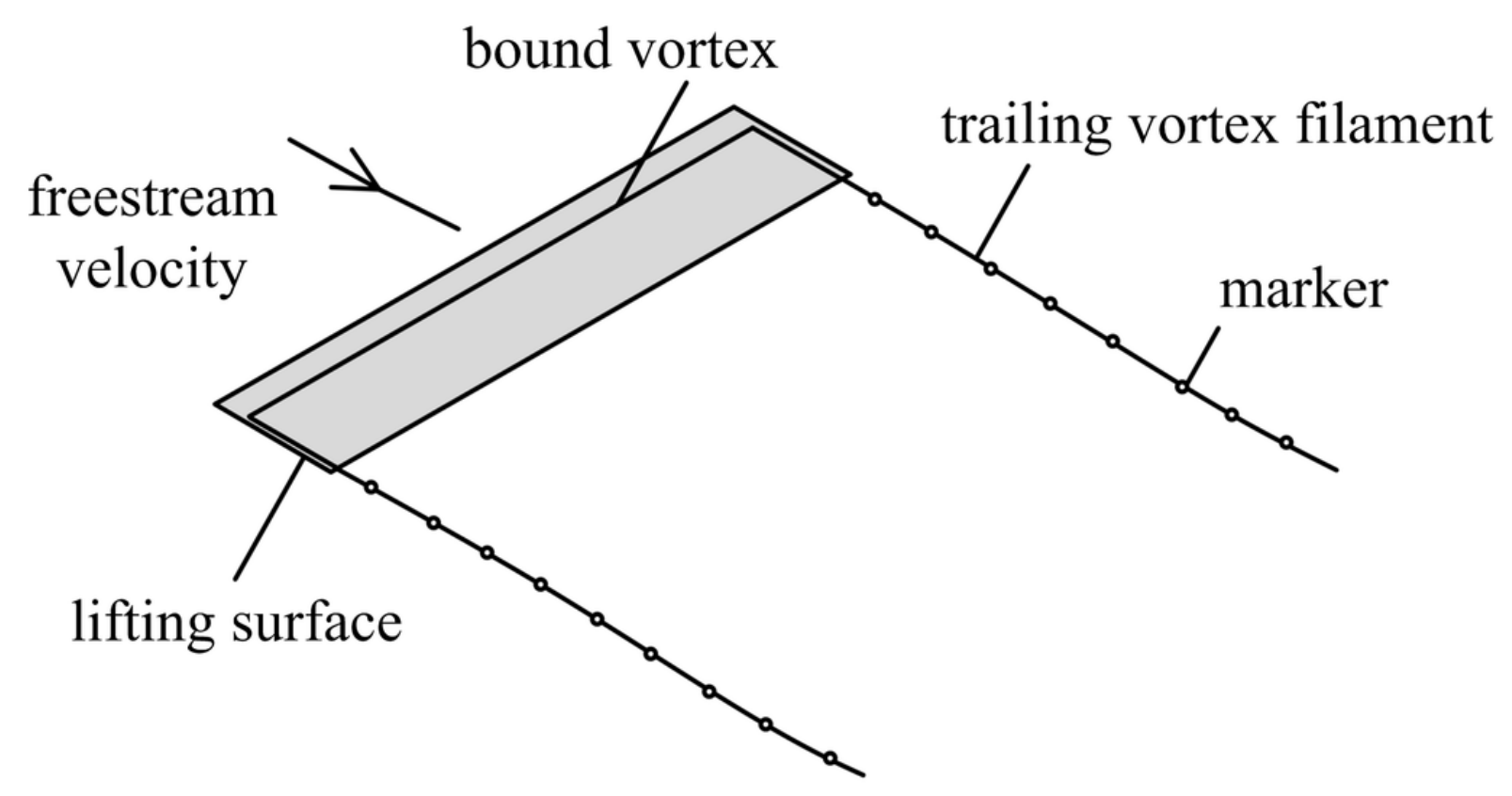

b)

c)
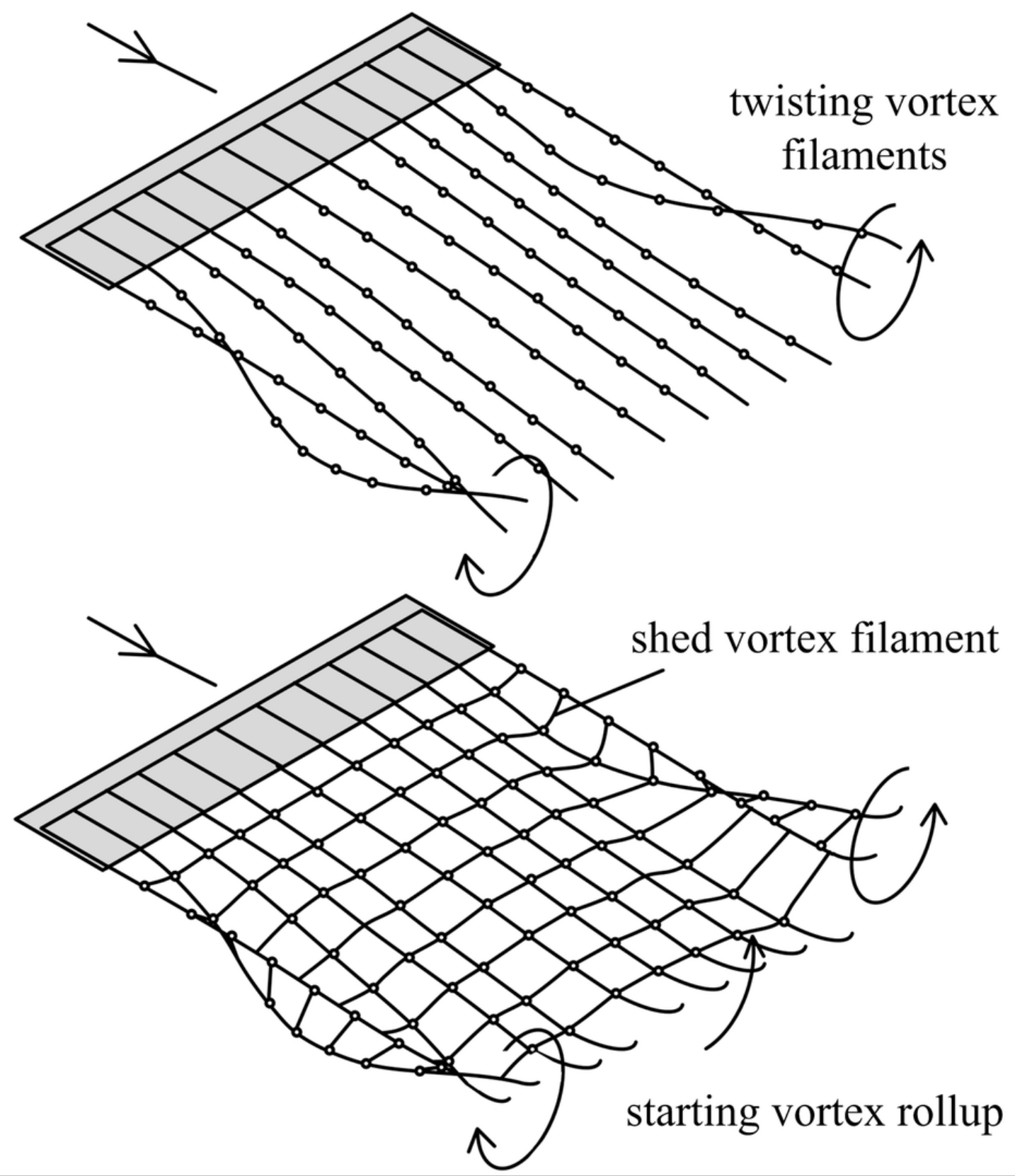


\section{Figure 2}

Illustration of vortex rings being shed from a lifting surface

(a) Lagrangian markers define the ring locations. Circulation ( $\left.\Gamma_{1-6}\right)$ is summed for neighbouring vortex rings to give the net circulation on a segment between each marker pair. (b) Example of net circulation on four filament segments.

a)

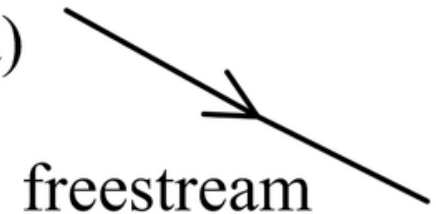

freestream velocity

b)
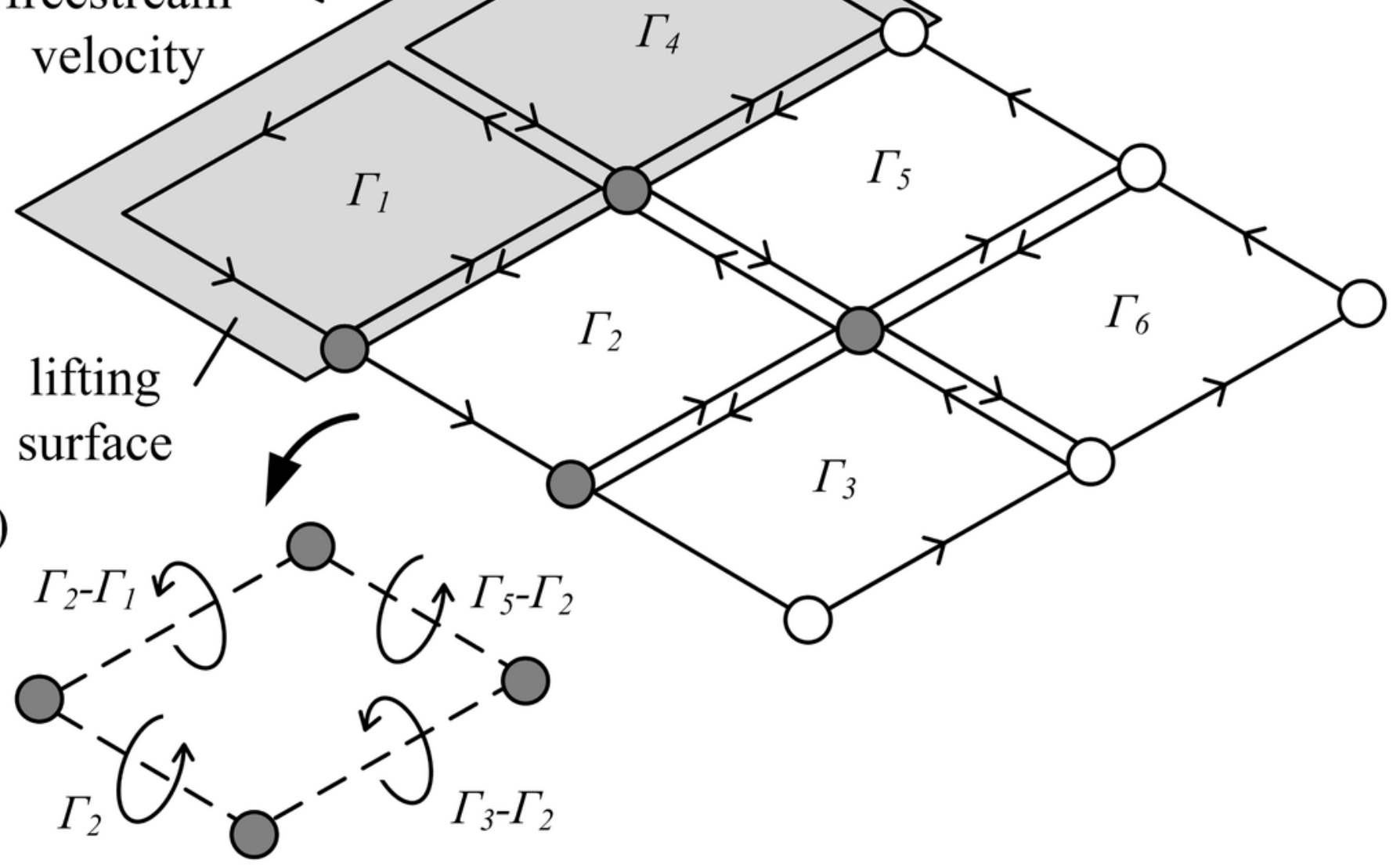


\section{Figure 3}

Simulated wakes for fixed rectangular wings in a $10 \mathrm{~ms}^{-1}$ flow; wing length and surface area are the same as those of the Rock Pigeon.

(a) The influence of the body is neglected; (b) the body is assumed to have the same lift per unit span as the wings. (c) Simulation from (b) with a cubic spline interpolation of the Lagrangian marker positions. 
a)

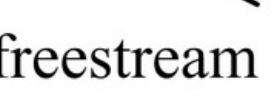

velocity

b)
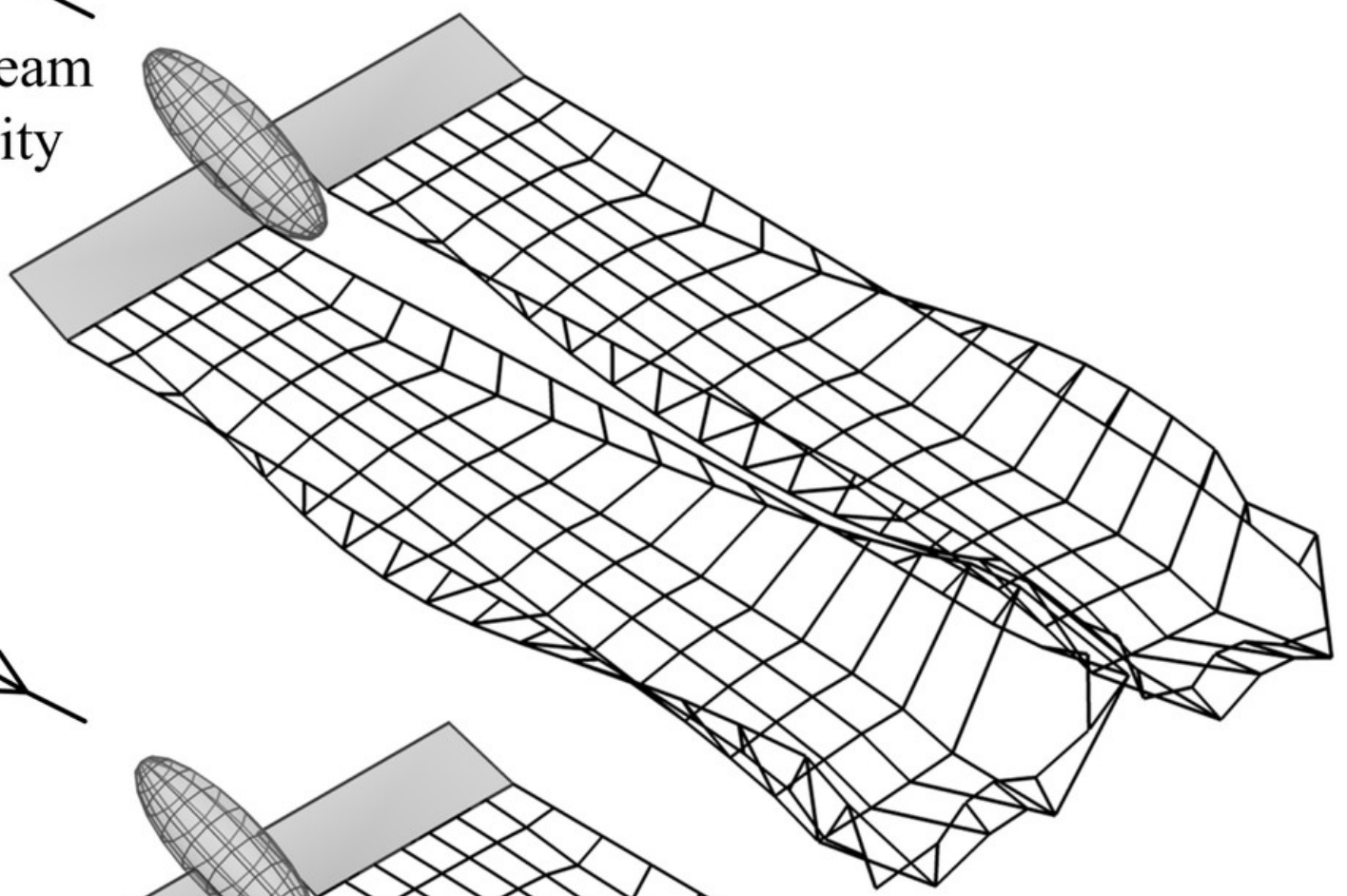

c)
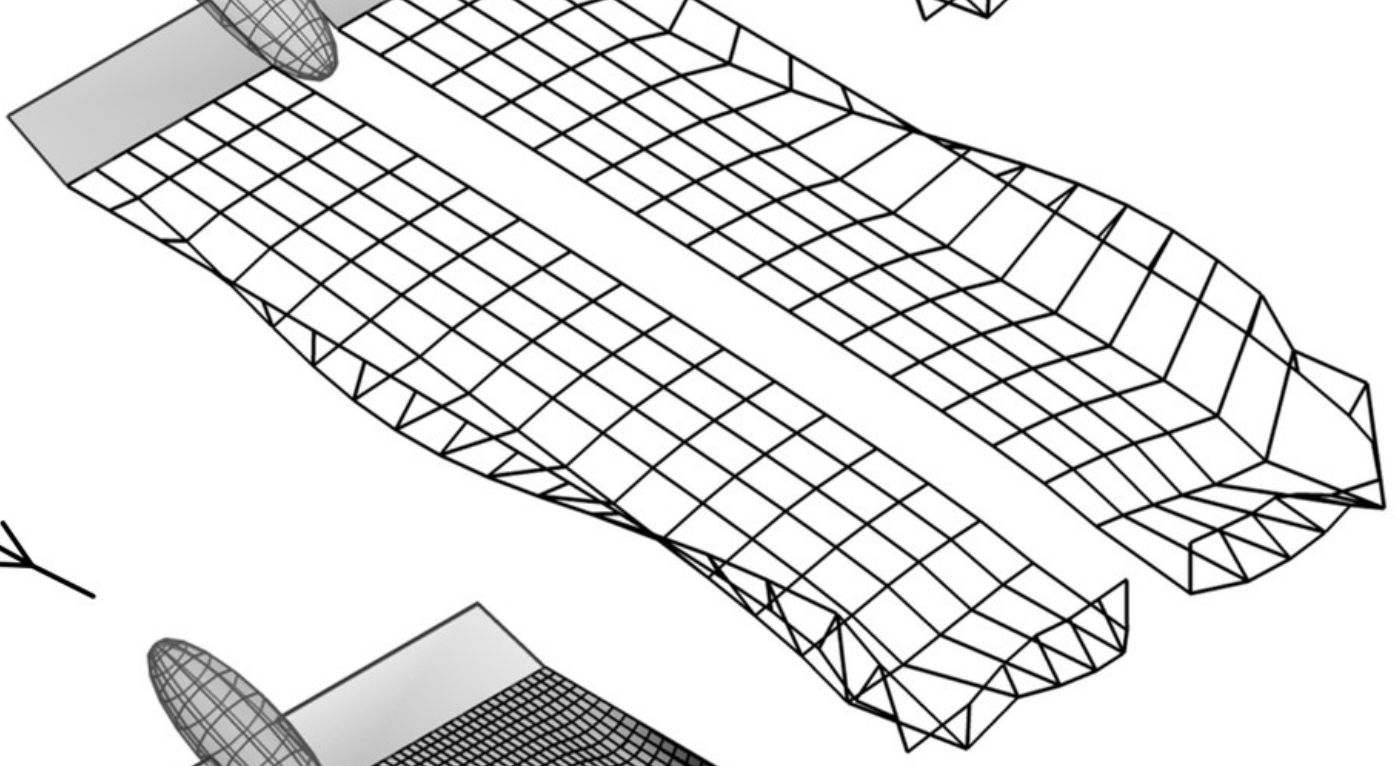


\section{Figure 4}

Numerical convergence study showing the variation in wake geometry with varying numbers of the wake parameters.

Wake parameters include the number of time steps per cycle, $n_{t}$, number of vortex filaments, $n_{f}$, and number of markers released per cycle for each filament, $n_{m}$. Simulations were conducted using wing dynamics predicted from models of the Rock Pigeon in minimum power cruising flight [11] . The wake was simulated for three wingbeats, and the marker positions were taken from markers released during the third wingbeat to avoid interference from the starting vortex.

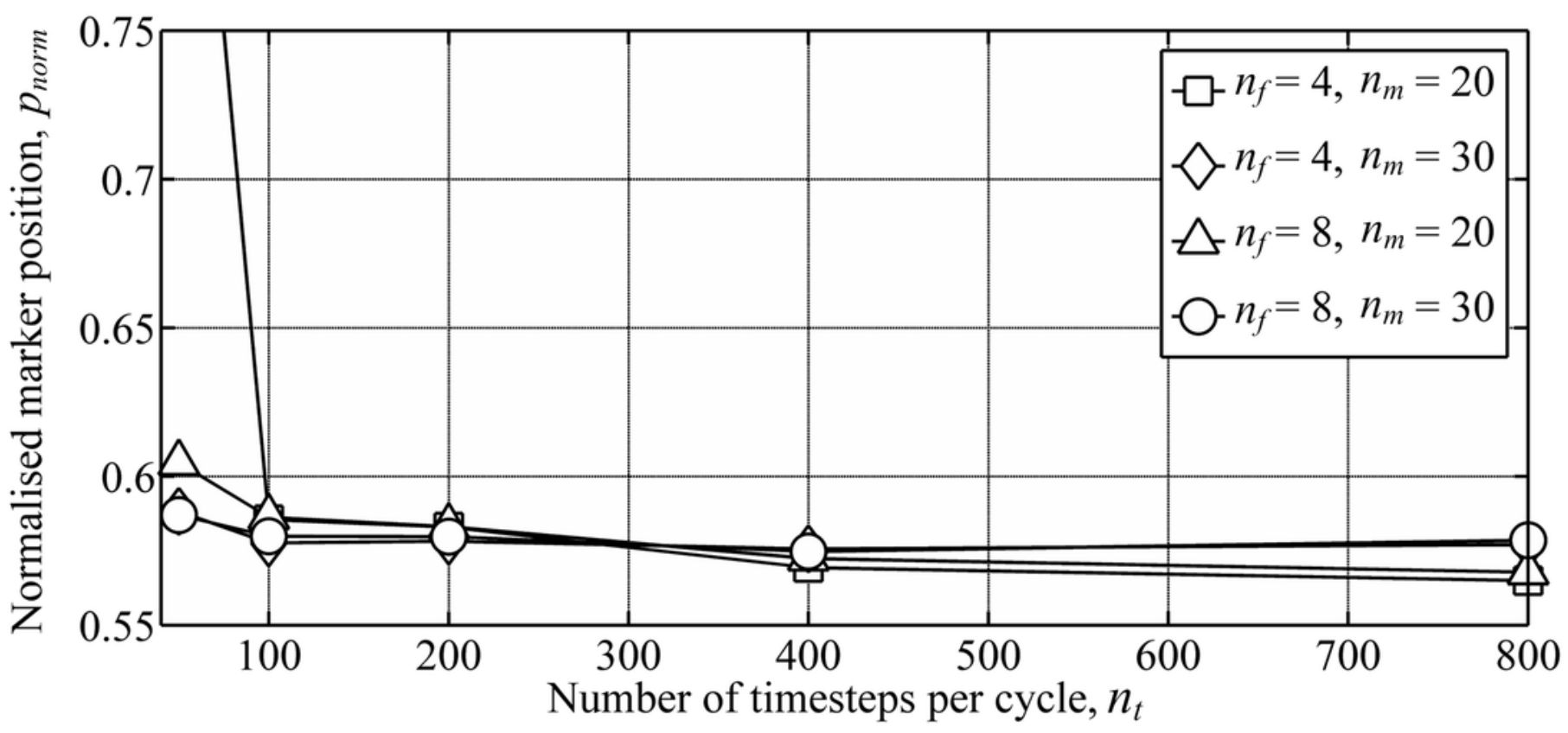




\section{Figure 5}

Wake geometry for simulated minimum power cruising flight of the Rock Pigeon over three wingbeats.

Wing dynamics were obtained using the model presented in [11] . Simulations were conducted using 800 time steps per cycle, 8 filaments per wing, and 30 markers released per cycle for each filament. Wake surfaces are shown from side (a) top (b) and isometric (c) perspectives; dot-dash line at $x / l_{w}=3.6$ indicates the streamwise location of the velocity plane used in (d), which shows the induced velocity predicted on a uniform grid of nodes when viewed in the negative $x$ direction, with a greyed projection of the bird body and wings.

a)

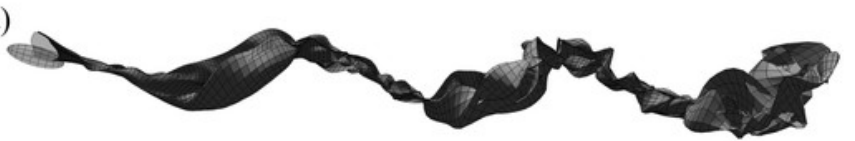

b)

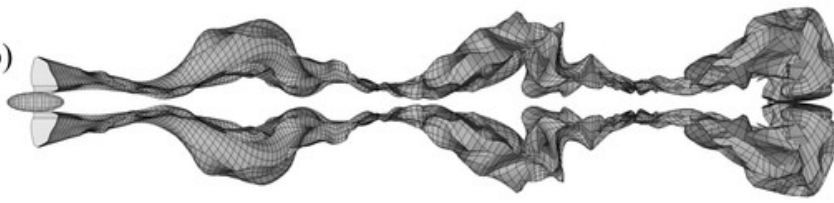

c)

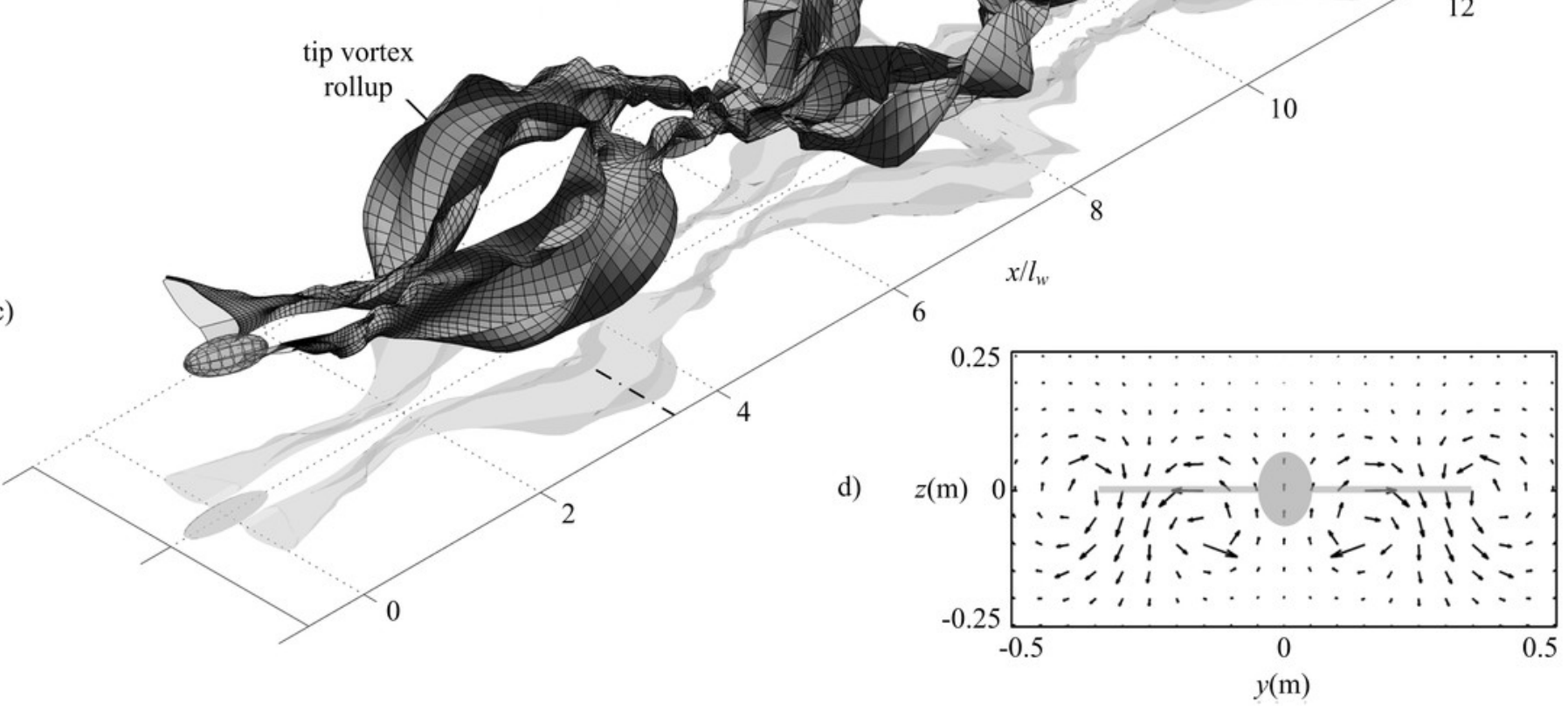

